TRANSACTIONS OF THE

AMERICAN MATHEMATICAL SOCIETY

Volume 354, Number 6, Pages 2359-2367

S 0002-9947(02)02979-3

Article electronically published on February 7, 2002

\title{
THREE-DIVISIBLE FAMILIES OF SKEW LINES ON A SMOOTH PROJECTIVE QUINTIC
}

\author{
SEAWOMIR RAMS
}

\begin{abstract}
We give an example of a family of 15 skew lines on a quintic such that its class is divisible by 3 . We study properties of the codes given by arrangements of disjoint lines on quintics.
\end{abstract}

\section{IntroduCtion}

The aim of this note is to study sets of lines on quintic surfaces $Y \subset \mathbb{P}_{3}(\mathbb{C})$. A divisor $\Lambda$ is said to be 3-divisible if its class in $\mathrm{NS}(Y)$ is divisible by 3 . If

$$
\Lambda=\sum_{1}^{p} L_{i}-\sum_{p+1}^{p+q} L_{i}
$$

with disjoint lines $L_{i} \subset Y$, is 3-divisible, we call it a $(p, q)$-divisor. In this note we give an example of a $(12,3)$-divisor (see Sect. 2) $)$. We prove bounds on the weight and the dimension of the code given by a $(p, q)$-divisor. We also collect restrictions on the numbers $(p, q)$, see Sect. 4 .

It is well-known that the maximal number of disjoint lines on a smooth quartic $X_{4}$ is $\left.16(10]\right)$. If a family of skew lines on $X_{4}$ is even (i.e. divisible by 2 ), then it consists of eight or 16 lines ([10]). Every family of 16 skew lines on a quartic is a sum of even families of eight lines, whereas a family of eight lines is even under a condition that involves the existence of a configuration of rational or elliptic curves ([1]).

In the case of a smooth quintic surface $Y$, it is not known whether the bounds [9] (at most 30 skew lines), [13] (at most 147 lines) are sharp. One can see, however, that a family of 30 skew lines on $Y$, if there is any, defines a $d$-dimensional $\mathbb{F}_{3}$-code with $d \geq 4$. Moreover, by the Griesmer bound, such a $Y$ must contain $(p, q)$-divisors with $p+q \leq 18$.

In this note we prove that the support of a $(p, q)$-divisor contains at least 15 lines, and that this bound is sharp. We give analogues of conditions 1 for a family of skew lines to be 3-divisible. We apply S.-L. Tan's generalization of Beauville's technique to prove that every $(p, q)$-divisor is a sum of $(r, s)$-divisors with $r, s \leq 15$. Finally, we show that a reduced 3-divisible family must consist of 15 lines, and compute invariants of the triple covers associated with $(p, q)$-divisors.

Received by the editors December 31, 2000.

2000 Mathematics Subject Classification. Primary 14M99; Secondary 14E20.

Key words and phrases. Quintic, cyclic cover, code.

This research was supported by DFG contract BA 423/8-1. 


\section{ThreE-DIVISIBLE DIVISORS WITH AT MOST 18 LINES}

Let $Y$ be a smooth quintic surface in $\mathbb{P}_{3}(\mathbb{C})$. In this note we study properties of the divisors

$$
\Lambda:=\sum_{1}^{p} L_{i}-\sum_{p+1}^{p+q} L_{i}=3 \mathcal{L},
$$

where the $L_{i}$ are pairwise disjoint lines on $Y$ and $\mathcal{L} \in \operatorname{Pic}(Y)$. The divisor $\Lambda$ will be called a $(p, q)$-divisor. We assume $p \geq q$.

Let $\mathcal{K}_{Y}$ be the canonical divisor of $Y$. We have the equalities

$$
\mathcal{L}^{2}=-\frac{p+q}{3}, \quad\left(\mathcal{K}_{Y}-\mathcal{L}\right)^{2}=5-p+\frac{q}{3} .
$$

Since $\operatorname{deg}(\mathcal{L})=\frac{1}{3}(p-q)$, both $p$ and $q$ are divisible by 3 . Moreover,

$$
\mathrm{h}^{0}(\mathcal{L})=0 \text { for every } p, q .
$$

Indeed, suppose that $\mathcal{L}$ is effective. Then, since $\mathcal{L} . L_{i}=-1$ for $i \leq p$, we have $\mathcal{L}=L_{1}+\ldots+L_{p}+D^{\prime}$, with an effective $D^{\prime}$, so $\operatorname{deg}(\mathcal{L}) \geq p$. Contradiction.

By Riemann-Roch we have $\chi(\mathcal{L})=5-\frac{p}{3}$, which implies that

$$
\mathcal{K}_{Y}-\mathcal{L}=\mathcal{O}(1)-\mathcal{L} \text { is effective for } p \leq 12,
$$

$$
\left.\mathcal{O}(1)-\mathcal{L} \text { is moving (i.e. } \mathrm{h}^{0}(\mathcal{O}(1)-\mathcal{L}) \geq 2\right) \text { for } p \leq 9 .
$$

Lemma 2.1. Let $D$ be an effective divisor on $Y$ with $D^{2}<4$.

a) If $\operatorname{deg}(D) \leq 4$, then $D$ is moving iff it is a plane quartic residual to a line $L_{0} \subset Y$. It moves by the pencil of planes through $L_{0}$. In particular, $D^{2}=0$.

b) If $\operatorname{deg}(D)=5$ and $D$ is moving, then the unique fixed component of $|D|$ is a line $L$, and the generic $D_{1} \in|D-L|$ is irreducible. Moreover, L. $D_{1}<2$.

Proof. a) An irreducible curve $\subset Y$ of degree $\leq 3$ has arithmetic genus $\leq 1$ and negative self-intersection on $Y$, a surface of general type. Hence if $D$ is moving, then $\operatorname{deg}(D)=4$, and the generic $C \in|D|$ is irreducible. If the generic $C \in|D|$ is singular, we find a base point at which all $C \in|D|$ have multiplicity $\geq 2$, which yields $D^{2} \geq 2^{2}$, and contradicts our assumption. By adjunction and Castelnuovo's inequality, the generic $C \in|D|$ is planar. Let $L_{0}$ be the line in $|\mathcal{O}(1)-C|$. Then $L_{0} . C=4$, which implies $|D|=\left|\mathcal{O}(1)-L_{0}\right|$.

b) If the generic $C \in|D|$ is irreducible, we can find a smooth quintic in $|D|$. By Castelnuovo's inequality the only smooth irreducible quintic $D \subset Y$ with nonnegative self-intersection is the planar one. Hence $|D|=\left|D_{1}\right|+L$, where $L$ is a line. By Bertini ([6, Lemma on p.536]), components of the generic $C \in\left|D_{1}\right|$ have non-negative self-intersection, and $C$ is irreducible.

If $L . D_{1} \geq 2$, we have $D_{1}^{2}<7-2 D_{1} . L<4$, so a) implies that $D_{1}$ is planar. Thus $D_{1} \in|\mathcal{O}(1)-L|$, and $D^{2}=5$. Contradiction.

Theorem 2.2. If a divisor of the type $(p, q)$ is 3 -divisible, then either $p \geq 15$, or $p=12$ and $q \geq 3$, or $p=q=9$.

Proof. Assume $0<p \leq 9$. Then (see (2.3) $D:=\mathcal{O}(1)-\mathcal{L}$ is a moving divisor of degree $5+\frac{1}{3}(q-p) \leq 5$ with $D^{2}<4$.

Suppose $\operatorname{deg}(D)=4$. Lemma 2.1 a implies $\mathcal{O}(1)-\mathcal{L}=\mathcal{O}(1)-L_{0}$, where $L_{0} \subset Y$ is a line. Hence $\mathcal{L}$ is effective, which contradicts (2.1). 
Assume $0<p=q \leq 6$ (i.e. $\operatorname{deg}(D)=5$ ). Let $L$ be the fixed component of $|D|$. Then $\left(\mathcal{L}+D_{1}\right) \cdot L=\left(\mathcal{K}_{Y}-L\right) . L=4$. Since $3 \mathcal{L} . L \leq 6$, we get $D_{1} . L \geq 2$. The latter cannot happen by Lemma $2.1 \mathrm{~b}$.

For $(p, q)=(12,0)$, we have $\operatorname{deg}(D)=1$, so $D$ is a line with $D^{2}=-7$.

Example 2.3. Consider the surface $S:=\mathrm{V}\left(X_{1}^{4} X_{2}+X_{2}^{4} X_{1}+X_{3}^{4} X_{4}+X_{4}^{4} X_{3}\right)$. We introduce the following notation:

$\omega$ (resp. $\varepsilon$ ) stands for a primitive root of unity of degree 3 (resp. 5 ),

$A_{i, j}$ denotes the line $\mathrm{V}\left(X_{i}, X_{j}\right)$, where $1 \leq i \leq 2<j \leq 4$,

$C_{l, s}$ is the line that passes through $\left(0: 1: 0:-\eta^{4}\right),(-\eta: 0: 1: 0)$, where

$\eta:=\omega^{l} \varepsilon^{s}$ and $0 \leq l \leq 2,0 \leq s \leq 4$,

$D_{l, s}$ is the line that passes through $\left(1: 0: 0:-\eta^{4}\right),(0:-\eta: 1: 0)$,

$Q_{s}$, where $0 \leq s \leq 4$, denotes the quadric $\mathrm{V}\left(X_{1} X_{2}-\varepsilon^{2 s} X_{3} X_{4}\right)$.

Observe that $Q_{s} \cap S$ consists of the lines $A_{i, j}, C_{l, s}, D_{l, s}$, where $l=0,1,2, i=$ $1,2, j=3,4$. Moreover, $C_{l, s}, A_{1,4}, A_{2,3}$ belong to one ruling of $Q_{s}$, whereas $D_{l, s}$, $A_{1,3}, A_{2,4}$ belong to the other one. Fix an $s_{0} \in\{0, \ldots, 4\}$ and define

$$
\Lambda:=\sum_{\substack{j=0, \ldots, 2 \\ s \neq s_{0}}} C_{j, s}-\sum_{j=0, \ldots, 2} D_{j, s_{0}} .
$$

Observe that $C_{l_{1}, s_{1}} \cap D_{l_{2}, s_{2}} \neq \emptyset$ iff $s_{1}=s_{2}$, which implies that $\operatorname{supp}(\Lambda)$ consists of 15 disjoint lines. Furthermore, $A_{1,3}, A_{2,4}$ meet the twelve lines $C_{l, s} \subset \operatorname{supp}(\Lambda)$, and do not meet the lines $D_{j, s_{0}}$.

One can prove that $S$ contains precisely 55 lines, and that the maximal number of pairwise disjoint lines on $S$ is 19 (e.g. the family

$$
C_{i, j}, \mathrm{~V}\left(X_{1}, X_{3}+\omega X_{4}\right), \mathrm{V}\left(X_{2}, X_{3}+X_{4}\right), \mathrm{V}\left(X_{3}, X_{1}+\omega X_{2}\right), \mathrm{V}\left(X_{4}, X_{1}+X_{2}\right)
$$

consists of 19 skew lines). Moreover, the only divisors supported by at least 15 skew lines which meet all of those 55 lines with multiplicities divisible by 3 are the ten divisors defined as $\Lambda$. The fact that $\Lambda$ is a $(12,3)$-divisor results from Lemma 2.4.

Lemma 2.4. Let $L_{1}, \ldots, L_{15}$ be a family of skew lines on a smooth projective quintic $Y$. Then the following conditions are equivalent:

(1) $3 \mid \sum_{1}^{12} L_{i}-\sum_{13}^{15} L_{i}$ in $\mathrm{H}^{2}(Y, \mathbb{Z})$,

(2) there exist disjoint lines $M_{1}, M_{2} \subset Y$ such that for $k=1,2$

$$
M_{k} \cap L_{i} \neq \emptyset \text { for } i \leq 12 \text {, and } M_{k} \cap L_{i}=\emptyset \text { for } i=13,14,15 \text {. }
$$

Moreover, in this case $M_{1}+M_{2} \in\left|\mathcal{K}_{Y}-\frac{1}{3}\left(\sum_{1}^{12} L_{i}-\sum_{13}^{15} L_{i}\right)\right|$.

Proof. "(1) $\Rightarrow(2)$ ": Put $D:=\mathcal{K}_{Y}-\mathcal{L}$, where $3 \mathcal{L}=\sum_{1}^{12} L_{i}-\sum_{13}^{15} L_{i}$. Since $D^{2}=$ -6 , the system $|D|$ contains no irreducible conic. As $\chi(\mathcal{L})=1$, the system $\left|\mathcal{K}_{Y}-\mathcal{L}\right|$ must contain a sum of disjoint lines $M_{1}+M_{2}$. Then D. $M_{k}=-3=1-\mathcal{L} . M_{k}$. Thus $3 \mathcal{L} \cdot M_{k}=12$, and $M_{k} . L_{i}=1$ iff $i \leq 12$.

"(2) $\Rightarrow(1) "$ : Put $F:=3 \mathcal{K}_{Y}-3\left(M_{1}+M_{2}\right)-\left(\sum_{1}^{12} L_{i}-\sum_{13}^{15} L_{i}\right)$. Then

$$
F^{2}=0 \text { and }\left(\mathcal{K}_{Y} \cdot F\right)=0
$$

imply $F=r \mathcal{K}_{Y}$, where $r \in \mathbb{Q}$, by the Hodge index theorem (recall that $\mathrm{H}^{2}(Y, \mathbb{Z})$ is torsion-free). But $0=F^{2}=5 r^{2}$ shows that $F=0$ in $\mathrm{H}^{2}(Y, \mathbb{Z})$.

Let $L_{1}, \ldots, L_{18}$ be a family of skew lines on a smooth quintic $Y$. 
Lemma 2.5. The following conditions are equivalent:

(1) $3 \mid \sum_{1}^{12} L_{i}-\sum_{13}^{18} L_{i}$ in $\mathrm{H}^{2}(Y, \mathbb{Z})$,

(2) there exists a smooth rational cubic $C \subset Y$ such that

$$
\text { C. } L_{i}=2 \text { for } i \leq 12 \text {, and } C \cap L_{i}=\emptyset \text { for } i>12 \text {. }
$$

Moreover, in this case $C \in\left|\mathcal{K}_{Y}-\frac{1}{3}\left(\sum_{1}^{12} L_{i}-\sum_{13}^{18} L_{i}\right)\right|$.

Proof. "(1) $\Rightarrow(2)$ ": Define $D$ and $\mathcal{L}$ as in the proof of Lemma 2.4 By the equality (2.2) the system $|D|$ contains a (possibly reducible) cubic $C$. Since $\mathrm{p}_{\mathrm{a}}(C)=0$ and $C . L_{i}=2$ (resp. $\left.C . L_{i}=0\right)$ for $i \leq 12$ (resp. $i>12$ ), it suffices to prove that $C$ is irreducible.

Suppose that the contrary holds. Since $D^{2}=-5$, we must have $C=C_{2}+L$, where $C_{2}$ (resp. $L$ ) is a (possibly singular) conic (resp. a line) and $L \cdot C_{2}=1$. Thus $\mathcal{L} . L=\left(\mathcal{K}_{Y}-C_{2}-L\right) . L=3$. Hence $C_{2} .3 \mathcal{L}=15$, and there exist two lines $L_{i}, L_{j}$ such that $L_{i} . C_{2}=L_{j} . C_{2}=2$. Contradiction.

"(2) $\Rightarrow(1) "$ : Apply the Hodge index theorem.

Lemma 2.6. The following conditions are equivalent:

(1) $3 \mid \sum_{1}^{9} L_{i}-\sum_{10}^{18} L_{i}$ in $\mathrm{H}^{2}(Y, \mathbb{Z})$,

(2) there exist disjoint lines $M_{1}, M_{2} \subset Y$ such that

$$
\begin{aligned}
& M_{1} \cap L_{i} \neq \emptyset \text { for } i \leq 9, \text { and } M_{1} \cap L_{i}=\emptyset \text { for } i>9, \\
& M_{2} \cap L_{i} \neq \emptyset \text { for } i>9, \text { and } M_{2} \cap L_{i}=\emptyset \text { for } i \leq 9 .
\end{aligned}
$$

Moreover, in this case $\sum_{1}^{9} L_{i}-\sum_{10}^{18} L_{i}=3\left(M_{2}-M_{1}\right)$ in $\mathrm{H}^{2}(Y, \mathbb{Z})$.

Proof. "(1) $\Rightarrow(2)$ ": Put $D_{1}:=\mathcal{K}_{Y}-\mathcal{L}, D_{2}:=\mathcal{K}_{Y}+\mathcal{L}$, where $3 \mathcal{L}=\sum_{1}^{9} L_{i}-\sum_{10}^{18} L_{i}$. By Lemma 2.1]b $\left|D_{k}\right|=\left|C_{k}\right|+M_{k}$, where $M_{k}$ is a line. Lemma 2.1] implies that $C_{k}$ is planar. Hence $C_{1} \cdot L_{i} \leq 1$. Since $D_{1} \cdot L_{i}=2$ (resp. $\left.D_{1} \cdot L_{i}=0\right)$ for $i \leq 9$ (resp. $i>9$ ), we get $M_{1} \cdot L_{i}=1$ (resp. $M_{1} \cdot L_{i}=0$ ) when $i \leq 9$ (resp. $i>9$ ). The proof for $M_{2}$ is analogous.

As $\left(C_{1}+M_{1}\right) \cdot M_{2}=1-\mathcal{L} \cdot M_{2}=4$ and $C_{1}$ is planar, we have $M_{1} \cdot M_{2}=0$.

"(2) $\Rightarrow(1) "$ : Define $F:=3\left(M_{2}-M_{1}\right)-\left(\sum_{1}^{9} L_{i}-\sum_{10}^{18} L_{i}\right)$ and apply the Hodge index theorem.

\section{Covers and Codes Given By $(p, q)$-Divisors}

Let us consider the mapping $\varphi: \mathbb{F}_{3}^{p+q} \rightarrow \operatorname{Pic}(Y) \otimes \mathbb{F}_{3}$ given by the formula

$$
\varphi: \sum_{1}^{p+q} \mu_{j} e_{j} \mapsto \sum_{1}^{p+q} \mu_{j} L_{j}
$$

Observe that every vector (word) in $\operatorname{ker}(\varphi)$ corresponds to an effective 3-divisible $\Lambda^{\prime} \subset \Lambda$ (we write $\Lambda^{\prime} \subset \Lambda$ instead of $\operatorname{supp}\left(\Lambda^{\prime}\right) \subset \operatorname{supp}(\Lambda)$ ).

In this section we prove a lower bound on $\operatorname{dim}_{\mathbb{F}_{3}}(\operatorname{ker} \varphi)$.

Put $\mathcal{L}^{\prime}:=\mathcal{L}+\sum_{p+1}^{p+q} L_{i}$. Riemann-Roch yields that

$$
\chi\left(-\mathcal{L}^{\prime}\right)=5-\frac{q}{3} \text {, and } \chi\left(-2 \mathcal{L}^{\prime}\right)=5-\frac{p}{3}-2 q .
$$

The bundle $\mathcal{L}^{\prime}$ defines a $3: 1$ cyclic cover $Z \rightarrow Y$ branched over the lines $L_{i}$ (see [3, I. $\S 17])$. Its structure sheaf $\mathcal{O}_{Z}$ is the $\mathcal{O}_{Y}$-algebra

$$
\mathcal{O}_{Y} \oplus \mathcal{O}_{Y}\left(-\mathcal{L}^{\prime}\right) \oplus \mathcal{O}_{Y}\left(-2 \mathcal{L}^{\prime}\right) .
$$


The surface $Z$ is smooth over the lines $L_{i}$, where $i \leq p$. It is singular (with local equation $z^{3}=x^{2}$ ) over the lines $L_{i}$ for $i>p$. Let $\nu: X \rightarrow Z$ be the normalization of $Z$, and let $\tilde{L}_{i} \subset X$ be the (reduced) rational curve lying over $L_{i}$. Then $X$ is smooth and we have the exact normalization sequence

$$
0 \rightarrow \mathcal{O}_{Z} \rightarrow \mathcal{O}_{X} \rightarrow \bigoplus_{p+1}^{p+q} \mathrm{I}_{\tilde{L}_{i}} / \mathrm{I}_{\tilde{L}_{i}}^{2} \rightarrow 0
$$

where $\mathrm{I}_{\tilde{L}_{i}} / \mathrm{I}_{\tilde{L}_{i}}^{2}=\mathcal{O}_{\tilde{L}_{i}}(1)$ and $\chi\left(\mathrm{I}_{\tilde{L}_{i}} / \mathrm{I}_{\tilde{L}_{i}}^{2}\right)=2$. Hence $\chi\left(\mathcal{O}_{X}\right)=\chi\left(\mathcal{O}_{Z}\right)+2 q$. By (B.2) we have $\chi\left(\mathcal{O}_{X}\right)=15-\frac{p+q}{3}$.

\section{Lemma 3.1.}

$$
\mathrm{h}^{1}\left(\mathcal{O}_{X}\right) \geq \frac{p}{3}-5 .
$$

Proof. The sequence (3.3) implies that $\mathrm{h}^{1}\left(\mathcal{O}_{X}\right)=\mathrm{h}^{1}\left(\mathcal{O}_{Z}\right)-2 q$. But

$$
\mathrm{h}^{1}\left(\mathcal{O}_{Z}\right)=\mathrm{h}^{1}\left(\mathcal{O}_{Y}\right)+\mathrm{h}^{1}\left(-\mathcal{L}^{\prime}\right)+\mathrm{h}^{1}\left(-2 \mathcal{L}^{\prime}\right) \geq-\chi\left(-2 \mathcal{L}^{\prime}\right)=\frac{p}{3}+2 q-5 .
$$

Observe that $Z$ is given by the Galois triple cover data $\left(\mathcal{L}, \mathcal{L}^{2}, 1, c\right)$ (see [8] Remark 7.6]), where $1 \in \mathcal{O}_{Y}$ and $\mathrm{V}(c)=\sum_{1}^{p} L_{i}+2 \sum_{p+1}^{p+q} L_{i}$ (i.e. $\sum_{1}^{p} L_{i}+2 \sum_{p+1}^{p+q} L_{i}$ is the divisor of zeroes of $\left.c \in \mathrm{H}^{0}\left(\mathcal{L}^{3}\right)\right)$. By [8, Prop. 7.5] $X$ is given by $(\mathcal{L}, \tilde{M}, \tilde{b}, \tilde{c})$, where $\tilde{M} \in \operatorname{Pic}(Y), \mathrm{V}(\tilde{b})=\sum_{p+1}^{p+q} L_{i}$, and $\mathrm{V}(\tilde{c})=\sum_{1}^{p} L_{i}$.

We will need the following version of [7, Lemma 3.2.1]:

\section{Lemma 3.2.}

$$
\operatorname{dim}_{\mathbb{F}_{3}}(\operatorname{ker} \varphi) \geq 1+h^{1}\left(\mathcal{O}_{X}\right) .
$$

Proof ([7]). Let $\operatorname{Tor}_{3}(X)$ denote the group of 3-torsions in $\operatorname{Pic}(X)$ and let $\operatorname{Tor}_{3}(X)^{G}$ stand for its subgroup of divisors invariant under the action of the cyclic group $G$ of the automorphisms of the cover. One can imitate the proof of [4. Lemma 2] (see [7, Lemma 1.2.1] for details) to construct an isomorphism

$$
\operatorname{Tor}_{3}(X)^{G} \simeq \operatorname{ker} \varphi / \mathbb{F}_{3} e,
$$

where $e=\sum_{1}^{p} e_{i}+\sum_{p+1}^{p+q} 2 e_{i} \in \mathbb{F}_{3}^{p+q}$. This yields the equality

$$
\operatorname{dim}_{\mathbb{F}_{3}}(\operatorname{ker} \varphi)=1+\operatorname{dim}_{\mathbb{F}_{3}}\left(\operatorname{Tor}_{3}(X)^{G}\right) .
$$

7, Cor. 1.2.3] gives the inequality $\operatorname{dim}_{\mathbb{F}_{3}}\left(\operatorname{Tor}_{3}(X)^{G}\right) \geq \frac{1}{2} \operatorname{dim}_{\mathbb{F}_{3}}\left(\operatorname{Tor}_{3}(X)\right)$.

(To prove that inequality, define $\beta: \operatorname{Pic}(X) \rightarrow \operatorname{Pic}(X)$ by $\beta(\eta)=\sigma^{*} \eta-\eta$, where $\sigma \neq \mathrm{id}, \sigma \in G$. We have the exact sequence

$$
0 \longrightarrow \operatorname{Tor}_{3}(X)^{G} \longrightarrow \operatorname{Tor}_{3}(X) \stackrel{\beta}{\longrightarrow} \operatorname{Im}(\beta) \longrightarrow 0 .
$$

But the sequence $0 \longrightarrow \operatorname{Tor}_{3}(X)^{G} \cap \operatorname{Im}(\beta) \longrightarrow \operatorname{Im}(\beta) \stackrel{\beta}{\longrightarrow} \operatorname{Im}\left(\beta^{2}\right)$ is exact and $\operatorname{Im}\left(\beta^{2}\right) \subset \pi^{*} \operatorname{Tor}_{3}(Y)=0$, where $\pi: X \rightarrow Z \rightarrow Y$ is the cover.)

The inequality $\operatorname{dim}_{\mathbb{F}_{3}}\left(\operatorname{Tor}_{3}(X)\right) \geq 2 \cdot \mathrm{h}^{1}\left(\mathcal{O}_{X}\right)$ concludes the proof.

Corollary 3.3. For every $(p, q)$-divisor, the following inequality holds:

$$
\operatorname{dim}_{\mathbb{F}_{3}}(\operatorname{ker} \varphi) \geq \frac{p}{3}-4 \text {. }
$$


Theorem 3.4. Every $(p, q)$-divisor $\Lambda$, where $p>15$ or $q>15$, is a sum of $\left(r_{i}, s_{i}\right)$ divisors $\Lambda_{i}$, with both $r_{i}, s_{i} \leq 15$.

\section{Reduced $(p, q)$-Divisors}

For $v \in \mathbb{F}_{3}^{n}$, one defines its weight $\mathrm{w}(v)$ as the number of its non-zero coordinates. An $[n, d, r]$-code is a $d$-dimensional subspace of $\mathbb{F}_{3}^{n}$ such that $r$ is the minimum of weights of its elements. We have the Griesmer bound ([11])

$$
n \geq \sum_{i=0}^{i=d-1}\left\lceil\frac{r}{3^{i}}\right\rceil
$$

Lemma 4.1. There are no $(p, 0)$-divisors for $p=18, \ldots, 24$.

Proof. Fix a $(p, 0)$-divisor $\Lambda$, where $18 \leq p \leq 24$, and consider the map $\varphi$ (see (3.1)). By Cor. 3.3 $\operatorname{ker}(\varphi)$ is a $[p, d, r]$-code with $d \geq 2$.

Suppose that $\Lambda_{1} \subset \Lambda$, where $\Lambda_{1}$ is a $(15-s, s)$-divisor and $s \leq 3$. Then $\Lambda-\Lambda_{1}$ is a $(p-15, s)$-divisor. Thm. 2.2 yields that $p \geq 27$. Thus $r \geq 18$.

By (4.1) $\Lambda$ is a $(24,0)$-divisor, and it must contain an $(18-s, s)$-divisor $\Lambda_{2}$ with $s \leq 9$. Then $\Lambda-\Lambda_{2}$ is a $(6, s)$-divisor with $s \leq 9$. Contradiction.

Remark 4.2. Let $\Lambda$ be an $(18,3)$-divisor. One can see that it contains no $(15-s, s)$ divisors. Hence, by (4.1), there are no (18,3)-divisors.

Lemma 4.3. There are no $(27,0)$-divisors.

Proof. Let $3 \mid \Lambda$, where $\Lambda:=\sum_{1}^{27} L_{i}$. Thm 2.2 implies that $\Lambda$ can contain only $(9,9),(12,12),(12,3)$ and $(3,12)$ divisors. By Cor. 3.3 and (4.1) it contains a $(12,3)$-divisor. We can assume that $\sum_{1}^{12} L_{i}-\sum_{24}^{27} L_{i}=3 \mathcal{L}_{1}$ in $\operatorname{Pic}(Y)$. Then

$$
\Lambda=3 \mathcal{L}_{1}+3 \mathcal{L}_{2}+3 \sum_{24}^{27} L_{i}
$$

where $3 \mathcal{L}_{2}=\sum_{13}^{24} L_{i}-\sum_{24}^{27} L_{i}$. Since $\left(\mathcal{K}_{Y}-\mathcal{L}_{1}\right) .\left(\mathcal{K}_{Y}-\mathcal{L}_{2}\right)=-2$, by Lemma 2.4 there exist lines $M_{1}, M_{2}, M_{3}$ such that $M_{k}+M_{3} \in\left|\mathcal{K}_{Y}-\mathcal{L}_{k}\right|$ for $k=1,2 . M_{3}$ meets $L_{i}$ iff $i \leq 24$, so $\left(M_{1}+M_{3}\right) \cdot \mathcal{L}_{2}=4$ gives $M_{1} \cdot L_{i}=0$ for $i>12$.

Claim 1. $\{v \in \operatorname{ker}(\varphi) \mid w(v) \neq 18\}=\operatorname{span}\left\{3 \mathcal{L}_{1}, 3 \mathcal{L}_{2}\right\}$.

Proof. Suppose $3 \mathcal{L}_{3} \subset \Lambda$, where $3 \mathcal{L}_{3}$ is a $(12,3)$-divisor in which precisely $\hat{p}$ (resp. $\hat{q}$ ) lines $L_{25}, L_{26}, L_{27}$ appear with multiplicity 1 (resp. -1$)$. Observe that $3 \mid(\hat{p}-\hat{q})=$ $9-3 \mathcal{L}_{3} \cdot M_{3}$. Since

$$
\left(\left(\mathcal{K}_{Y}-\mathcal{L}_{1}\right)+\left(\mathcal{K}_{Y}-\mathcal{L}_{2}\right)\right) \cdot\left(\mathcal{K}_{Y}-\mathcal{L}_{3}\right)=-5+(\hat{p}-\hat{q})<0,
$$

we can assume that $\left(\mathcal{K}_{Y}-\mathcal{L}_{1}\right) \cdot\left(\mathcal{K}_{Y}-\mathcal{L}_{3}\right)<0$. If $C \in\left|\mathcal{K}_{Y}-\mathcal{L}_{3}\right|$, then $C$ contains either $M_{1}$ or $M_{3}$, so $\hat{p}=0$.

In the first case, we have $3 \mathcal{L}_{3}=\sum_{1}^{12} L_{i}-\sum_{k=1}^{3} L_{j_{k}}$, so either $\mathcal{L}_{3}=\mathcal{L}_{1}$ (i.e. $\hat{q}=3)$ or all $j_{k} \leq 24(\hat{q}=0)$. If the latter holds, then $\left(\mathcal{K}_{Y}-\mathcal{L}_{1}\right) \cdot\left(\mathcal{K}_{Y}-\mathcal{L}_{3}\right)=-5$, which contradicts Lemma 2.4

Suppose that $M_{3} \subset C$. We have $3 \mathcal{L}_{3}=\sum_{k=1}^{12} L_{j_{k}}-\sum_{25}^{27} L_{i}$ with $j_{k} \leq 24$. Then we can assume $j_{k} \leq 12$ for $k \leq 6$, whence $\mathcal{L}_{1} \cdot \mathcal{L}_{3} \leq-3$, so $\left(\mathcal{K}_{Y}-\mathcal{L}_{1}\right) \cdot\left(\mathcal{K}_{Y}-\mathcal{L}_{3}\right)<-3$. Lemma 2.4 implies $\mathcal{L}_{1}=\mathcal{L}_{3}$.

If $\Lambda_{1} \subset \Lambda$ is a $(12,12)$-divisor, then $\Lambda-\Lambda_{1}$ is a $(3,12)$-divisor. Thus $\pm\left(3 \mathcal{L}_{1}-3 \mathcal{L}_{2}\right)$ are the only $(12,12)$-divisors $\subset \Lambda$. 
Claim 2. In every $(9,9)$-divisor precisely one of the lines $L_{25}, L_{26}, L_{27}$ appears with multiplicity 1 , and precisely one with multiplicity -1 .

Proof. Let $\sum_{k=1}^{9} L_{l_{k}}-\sum_{k=10}^{18} L_{l_{k}}=3\left(N_{2}-N_{1}\right)$, where $l_{k}$ is a permutation and $N_{1}, N_{2}$ are lines. Put $\mathcal{L}_{3}:=N_{2}-N_{1}, 3 \mathcal{L}_{4}:=\sum_{k=19}^{27} L_{l_{k}}-\sum_{k=1}^{9} L_{l_{k}}$, where $\mathcal{L}_{4} \in \operatorname{Pic}(Y)$. Then $\mathcal{L}_{3} \cdot \mathcal{L}_{4}=3$. Thus, by Lemma 2.6 there exists a line $N_{3} \subset Y$ such that $N_{3} \cdot\left(N_{1}+N_{2}\right)=0$ and $N_{3} \cdot L_{l_{k}}=1$ iff $k>18$. Moreover, $N_{1} \cdot L_{l_{k}}=1$ (resp. $\left.N_{2} \cdot L_{l_{k}}=1\right)$ iff $k<9$ (resp. $9<k \leq 18$ ).

Let $p_{j}$ (resp. $q_{j}$ ) be the number of the lines $L_{i}$, where $i \leq 12$ (resp. $i>24$ ), that meet $N_{j}$. Assume that $q_{1} \geq q_{3} \geq q_{2}$. Suppose that $\left(q_{1}, q_{2}, q_{3}\right) \neq(1,1,1)$. Then $q_{1} \geq 2$ and $q_{2}=0$. Observe that $3 \mid\left(p_{1}-q_{1}\right)=3 \mathcal{L}_{1} \cdot N_{1}$.

If $p_{1}>0$, then $p_{1} \geq 2$, so at least two lines $L_{i}$ meet $N_{1}, M_{1}, M_{3}$. Hence $N_{1} \cdot\left(M_{1}+M_{3}\right)=0$, and $\left(\mathcal{K}_{Y}-\mathcal{L}_{1}\right) \cdot \mathcal{L}_{3}=\left(M_{1}+M_{3}\right) \cdot\left(N_{2}-N_{1}\right) \geq 0$. Since

$$
\left(\left(\mathcal{K}_{Y}-\mathcal{L}_{1}\right)+\left(\mathcal{K}_{Y}-\mathcal{L}_{2}\right)\right) \cdot \mathcal{L}_{3}=-\left(q_{1}-q_{2}\right) \leq-2,
$$

we have $\left(M_{2}+M_{3}\right) \cdot\left(N_{2}-N_{1}\right) \leq-2$, which contradicts $M_{3} \cdot N_{1}=0$. Therefore, $p_{1}=0$ and $q_{1}=3$.

Repeating the same reasoning for $L_{i}$, where $12<i \leq 24$, and $3 \mathcal{L}_{2}$, one gets $N_{1} \cdot L_{i}=0$ for $i \leq 24$. Contradiction.

Fix a $(9,9)$-divisor $3 \mathcal{L}_{3} \subset \Lambda$. Let $3 \mathcal{L}_{4} \subset \Lambda$ be a $(9,9)$-divisor. One can see that in the word given by one of the divisors $\pm 3 \mathcal{L}_{3} \pm 3 \mathcal{L}_{4}$ all the lines $L_{25}, L_{26}, L_{27}$ appear either with multiplicity 1 or with multiplicity 0 , so (by Claim 2 ) that word is not given by a $(9,9)$-divisor, and (by Claim 1 ) belongs to $\operatorname{span}\left\{3 \mathcal{L}_{1}, 3 \mathcal{L}_{2}\right\}$. Therefore, $3 \mathcal{L}_{4} \in \operatorname{span}\left\{3 \mathcal{L}_{1}, 3 \mathcal{L}_{2}, 3 \mathcal{L}_{3}\right\}$, so $\operatorname{dim}(\operatorname{ker}(\varphi))=3$, which contradicts Cor. 3.3.

Remark 4.4. Similar reasoning yields that there are no (21,3)-divisors.

Theorem 4.5. If a divisor of the type $(p, 0)$ is divisible by 3 , then $p=15$.

Proof. We are to prove that there are no (30,0)-divisors. Let $3 \mid \Lambda:=\sum_{1}^{30} L_{i}$.

Thm 2.2 implies that $\Lambda$ contains no $(p, q)$-divisors with $18 \leq p<30$. If $\Lambda_{1} \subset \Lambda$ is a $(12,6)$-divisor, then $\Lambda_{2}:=\Lambda-\Lambda_{1}$ is a $(12,6)$-divisor, and $\left(\mathcal{K}_{Y}-\frac{1}{3} \Lambda_{1}\right) \cdot\left(\mathcal{K}_{Y}-\frac{1}{3} \Lambda_{2}\right)=$ -1 , which contradicts Lemma 2.5

Every $(p, q)$-divisor $\Lambda_{1} \subset \Lambda$ defines the 3-divisible divisors $\Lambda \pm \Lambda_{1}$. Thus $\operatorname{ker}(\varphi)$ has the folowing word distribution (we omit $(0,0), \pm(30,0)$ ):

\begin{tabular}{|c|c|c|c|c|c|c|}
$\pm(15,0)$ & $\pm(12,3)$ & $(9,9)$ & $\pm(15,3)$ & $\pm(12,9)$ & $\pm(15,12)$ & $\pm(15,15)$ \\
\hline $4 \mathrm{~d}_{1}$ & $2 \mathrm{~d}_{2}$ & $2 \mathrm{~d}_{3}$ & $2 \mathrm{~d}_{2}$ & $4 \mathrm{~d}_{3}$ & $2 \mathrm{~d}_{2}$ & $2 \mathrm{~d}_{1}$
\end{tabular}

where $d_{1} \leq 1$ (because $(15,0)-(15,0)=(15-s, 15-s)$, and Thm 2.2 implies $s \leq 6$. Then $(15,0)+(15,0)=(30-2 s, s)$, so $s=0)$. Hence, by Cor. 3.3 .

$$
\left(d_{2}+d_{3}\right) \geq \frac{1}{6}\left(3^{6}-9\right)=120 .
$$

If $3 \mathcal{L}_{1} \neq 3 \mathcal{L}_{2} \subset \Lambda$ are $(9,9)$-divisors, where $\mathcal{L}_{1}, \mathcal{L}_{2}$ are the divisors described in Lemma 2.6 and $\operatorname{supp}\left(\mathcal{L}_{1}\right) \cap \operatorname{supp}\left(\mathcal{L}_{2}\right)$ contains a line, then one of the divisors $3 \mathcal{L}_{1} \pm 3 \mathcal{L}_{2}$ is a $(9-r, 9-r)$-divisor. Thus $r=0$, which contradicts Lemma 4.3

Let $3 \mathcal{L}_{1} \neq 3 \mathcal{L}_{2} \subset \Lambda$ be $(12,3)$-divisors, $C_{i} \in\left|\mathcal{K}_{Y}-\mathcal{L}_{i}\right|$, and let $C_{1} \cap C_{2}$ contain a line. Then, by Lemma 2.4 $\operatorname{supp}\left(3 \mathcal{L}_{1}\right) \cap \operatorname{supp}\left(3 \mathcal{L}_{2}\right)$ consists of either 3 or 6 lines. In both cases, $\Lambda$ contains a $(12,12)$-divisor $\Lambda_{3}$ (given by $3 \mathcal{L}_{1}-3 \mathcal{L}_{2}$, resp. $3 \mathcal{L}_{1}+3 \mathcal{L}_{2}$ ), so $\Lambda-\Lambda_{3}=(6,12)$. Contradiction.

Let $3 \mathcal{L}_{1}\left(\right.$ resp. $\left.3 \mathcal{L}_{2}\right) \subset \Lambda$ be a $(9,9)$-divisor (resp. $(12,3)$ ), where $\mathcal{L}_{1}:=N_{2}-N_{1}$ and $N_{1}, N_{2}$ are lines. Let $C \in\left|\mathcal{K}_{Y}-\mathcal{L}_{2}\right|$. If $\operatorname{supp}\left(\mathcal{L}_{1}\right) \cap C$ contains a line, then either 
$N_{1}$ or $N_{2}$ meets at least twelve lines $L_{i}$. By Lemma 2.6 there exists a (9,9)-divisor $\Lambda_{4}$ such that $3 \mathcal{L}_{1}-\Lambda_{4}$ is a $(3,3)$-divisor.

Hence (4.2) implies that $Y$ contains at least $2 \cdot 120+30=270$ lines. Contradiction (see [13, §.4], [5, p.28]).

Let $W$ be the surface obtained by blowing down the (-1)-curves $\tilde{L}_{i}$. Then

$$
\mathcal{K}_{W}^{2}=15+(p+q), \text { and } \mathrm{c}_{2}(W)=165-5(p+q) .
$$

One can check that a $(21-s, s)$-divisor $\Lambda$ contains no $(15-s, s)$-divisors, so $\operatorname{dim}_{\mathbb{F}_{3}}(\operatorname{ker} \varphi)=1$. We get the following table of invariants.

\begin{tabular}{|c||l|c|c|c|c|}
\hline$p+q$ & $(p, q)$ & $\mathrm{h}^{1}\left(\mathcal{O}_{W}\right)$ & $\mathrm{p}_{\mathrm{g}}(W)$ & $\mathrm{c}_{1}^{2}(W)$ & $\mathrm{c}_{2}(W)$ \\
\hline \hline 15 & $(12,3),(15,0)$ & 0 & 9 & 30 & 90 \\
18 & $(9,9),(12,6),(15,3)$ & 0 & 8 & 33 & 75 \\
21 & $(12,9),(15,6)$ & 0 & 7 & 36 & 60 \\
\hline
\end{tabular}

For $p+q \geq 24$, Lemma 3.2 no longer implies $\mathrm{h}^{1}\left(\mathcal{O}_{W}\right)=0$. Observe that for $p+q=30$, the equality $\mathrm{c}_{1}^{2}(W)=3 \mathrm{c}_{2}(W)$ holds.

\begin{tabular}{|c||c|c|c|}
\hline$p+q$ & \multicolumn{1}{|c|}{$(p, q)$-divisors } & $\mathrm{c}_{1}^{2}(W)$ & $\mathrm{c}_{2}(W)$ \\
\hline \hline 24 & $(12,12),(15,9),(18,6)$ & 39 & 45 \\
\hline 27 & $(15,12),(18,9),(21,6),(24,3)$ & 42 & 30 \\
\hline 30 & $(15,15),(18,12),(21,9),(24,6),(27,3)$ & 45 & 15 \\
\hline
\end{tabular}

\section{ACKNOWLEDGEMENT}

I would like to express my sincere gratitude to Professor W. Barth for his constant help and numerous valuable discussions. I thank Professor D. van Straten for the script [12.

\section{REFERENCES}

[1] W. Barth: Even sets of eight skew lines on a K3 surface, preprint.

[2] W. Barth, I. Nieto: Abelian surfaces of type $(1,3)$ and quartic surfaces with 16 skew lines. J. Alg. Geom. 3 (1994), 173-222. MR 95e:14033

[3] W. Barth, A. Peters, A. Van de Ven: Compact Complex Surfaces. Berlin, Heidelberg, New York: Springer, 1984. MR 86c:32026

[4] A. Beauville: Sur le nombre maximum de points doubles d'une surface dans $\mathbb{P}^{3}(\mu(5)=31)$. in Journées de géometrie algebrique d'Angers (1979), Sijthoff-Noordhoff, 1980, pp. 207-215. MR 82k:14037

[5] L. Caporaso, J. Harris, B. Mazur: How many rational points can a curve have? in The Moduli Space of Curves (R. Dijkgraaf, C Faber, G. van der Geer eds.), Progress in Math. 129, Birkhäuser Verlag, 1995, pp. 13-31. MR 97d:11099

[6] P. Griffiths, J. Harris: Principles of algebraic geometry. New York, Chichester, Brisbane, Toronto, Singapore, John Wiley and Sons, Inc., 1978. MR 80b:14001

[7] Sheng-Li Tan: Cusps on some algebraic surfaces, preprint, 1999.

[8] R. Miranda: On triple covers in algebraic geometry Amer. J. Math. 107 (1985), 1123-1158. MR 86k:14008

[9] Y. Miyaoka: The Maximal Number of Quotient Singularities on Surfaces with Given Numerical Invariants, Math. Ann. 268 (1984) , 159-171. MR 85j:14060

[10] V.V. Nikulin: On Kummer Surfaces, Math. USSR. Izv. 9 (1975), no. 2, 261-275. MR 55:2926

[11] J.H. van Lint: Introduction to Coding Theory. Berlin: Springer, 1992. MR 94b:94001 
[12] D. van Straten: Macaulay script to estimate the number of lines on a surface with some examples of surfaces.

[13] B. Segre: The maximum number of lines lying on a quartic surface, Quart. J. Math. Oxford Ser. 14 (1943), 86-96. MR 6:16g

Institute of Mathematics, Jagiellon University, Reymonta 4, PL-30-059 Kraków, POLAND

Current address: Mathematisches Institut, FAU Erlangen-Nürnberg, Bismarckstrasse 1 1/2, D-91054 Erlangen, Germany

E-mail address: rams@mi.uni-erlangen.de and rams@im.uj.edu.pl 